\title{
Rotational thromboelastometry as a powerful tool to detect hyperfibrinolysis in a bleeding patient: a case report
}

\author{
KM Kwok ${ }^{1}$ *, FHKCP, FHKAM (Medicine), KL Lee ${ }^{1}$, FHKCP, FHKAM (Medicine), SY Lam¹, FHKCP, FHKAM (Medicine), \\ T Liong ${ }^{1}$, FHKCP, FHKAM (Medicine), HM Wong ${ }^{1}$, FHKCP, FHKAM (Medicine), PY Lam ${ }^{1}$, MBBS, MRCP, \\ Eudora Y Chow ${ }^{2}$, MBBS, FHKAM (Pathology), KI Law ${ }^{1}$, FHKCP, FHKAM (Medicine) \\ ${ }^{1}$ Intensive Care Department, United Christian Hospital, Hong Kong \\ ${ }_{2}^{2}$ Pathology Department, United Christian Hospital, Hong Kong \\ *Corresponding author: kkm394@ha.org.hk
}

Hong Kong Med J 2021;27:370-2

https://doi.org/10.12809/hkmj208971

\section{Case report}

A 64-year-old man presented to the emergency department with acute onset abdominal pain. His medical history was unremarkable. Initial vital signs showed blood pressure of $87 / 43 \mathrm{mmHg}$ and pulse rate of 110 beats per minute. His abdomen was distended, with generalised tenderness and guarding. The patient was stabilised with $1 \mathrm{~L}$ of crystalloid. Bedside ultrasonographic examination revealed intraperitoneal free fluid. Subsequent computed tomography of the abdomen demonstrated gross haemoperitoneum and multiple hepatic lesions highly suspicious of hepatocellular carcinoma (HCC). Active contrast extravasation was noted at the posterior aspect of segment $2 / 3$ lesion, compatible with the diagnosis of ruptured HCC. The initial haemoglobin level was $8.7 \mathrm{~g} / \mathrm{dL}$. Transarterial embolisation was urgently arranged but the patient went into cardiac arrest. Spontaneous circulation returned after 8 minutes of cardiopulmonary resuscitation. Haemoglobin level fell to $5.5 \mathrm{~g} / \mathrm{dL}$ and platelet count was normal. Prothrombin time and activated partial thromboplastin time were prolonged to $20.3 \mathrm{~s}$ and $62.1 \mathrm{~s}$, respectively. International normalised ratio was 1.9 . He was transfused with 2 units of pack cells and 4 units of plasma. Transarterial embolisation was successfully performed. Active contrast extravasation over a branch of the left hepatic artery was demonstrated but controlled by Gelfoam injection.

The patient was then transferred to the intensive care unit. Rotational thromboelastometry (ROTEM $^{\circledR}$; Tem International $\mathrm{GmbH}$, Munich, Germany) was performed to guide transfusion strategy (Fig a). Maximum lysis was shown to be $100 \%$, indicating abnormally accelerated clot lysis. Maximum lysis over $15 \%$ is diagnostic of hyperfibrinolysis. The lysis index at 30 minutes was $5 \%$, indicating almost complete clot dissolution 30 minutes after initial formation. No dysfunction in coagulation activation or clot propagation was otherwise detected. Tranexamic acid $1 \mathrm{~g}$ was administrated according to the interpretation of ROTEM results. A follow-up ROTEM analysis after antifibrinolytic treatment demonstrated restoration of normal fibrinolysis (Fig b). Haemostasis was achieved and no further blood transfusion was needed.

Testing for hepatitis B surface antigen was later revealed to be reactive. This patient probably had liver cirrhosis and $\mathrm{HCC}$ consequent to chronic hepatitis B infection. The occurrence of ruptured HCC tipped the balance in this vulnerable patient. He developed abdominal compartment syndrome and hepatic failure. The sequential organ failure assessment score was 13 with an estimated mortality of over $90 \%$. The surgical team advised optimum medical supportive treatment in view of the extremely high operative risk. The patient succumbed 16 hours after hospital admission.

\section{Discussion}

Haemocoagulation is a complex interaction of procoagulants, anticoagulants, fibrinolytic proteins, and cellular components. Platelets are activated in response to vascular injury, leading to primary haemostasis. Activated platelets adhere to damaged endothelium and aggregate to create a temporary platelet plug. Secondary haemostasis involves activation of the coagulation cascade, resulting in fibrin formation. The platelet plug is strengthened by the cross-linked fibrin to form a stable clot. Fibrinolysis serves as the final stage of coagulation to regulate the extent of clot formation and to maintain vascular patency. Cross-linked fibrin is broken down by plasmin and normal blood flow is restored.

Hyperfibrinolysis refers to excessive fibrinolytic activity that threatens clot integrity and leads to defective haemostasis. It is common in patients with chronic liver disease, major trauma, and obstetric complications. However, the incidence is not well studied and it has often been 
(a)

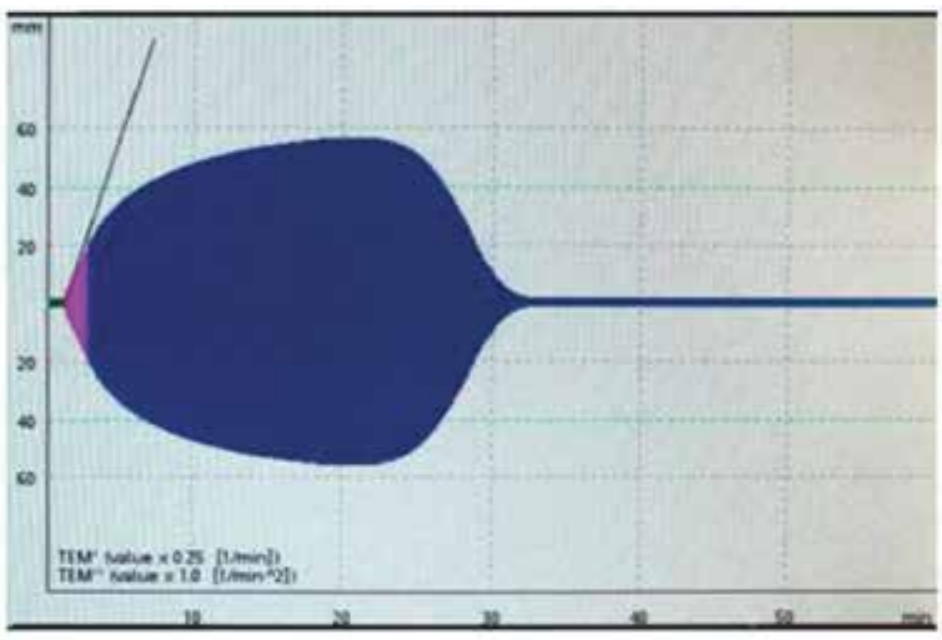

\begin{tabular}{|l|c|c|c|}
\hline EXTEM & \multicolumn{3}{|c|}{$2020-08-2410: 52$} \\
\hline CT & 76 & $\mathrm{~s}$ & $38-79$ \\
\hline CFT & 96 & $\mathrm{~s}$ & $34-159$ \\
\hline$\alpha$ & 76 & $\circ$ & $63-83$ \\
\hline A5 & 39 & $\mathrm{~mm}$ & $34-55$ \\
\hline AI0 & 50 & $\mathrm{~mm}$ & $43-65$ \\
\hline MCF & 56 & $\mathrm{Mm}$ & $50-72$ \\
\hline ML & 100 & $\%$ & $<15$ \\
\hline LI30 & 5 & $\%$ & $94-100$ \\
\hline
\end{tabular}

(b)

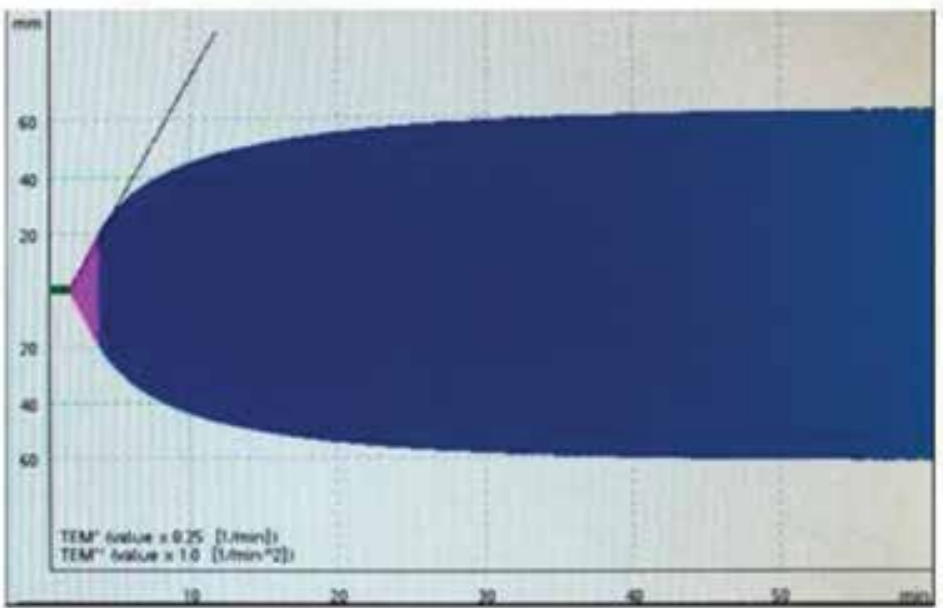

\begin{tabular}{|l|c|c|c|}
\hline EXTEM & \multicolumn{3}{|c|}{$2020-08-24$ I4:20 } \\
\hline CT & 100 & $\mathrm{~s}$ & $38-79$ \\
\hline CFT & 115 & $\mathrm{~s}$ & $34-159$ \\
\hline$\alpha$ & 67 & $\circ$ & $63-83$ \\
\hline A5 & 37 & $\mathrm{~mm}$ & $34-55$ \\
\hline A10 & 47 & $\mathrm{~mm}$ & $43-65$ \\
\hline MCF & 61 & $\mathrm{Mm}$ & $50-72$ \\
\hline ML & 1 & $\%$ & $<15$ \\
\hline LI30 & 100 & $\%$ & $94-100$ \\
\hline
\end{tabular}

FIG. (a) Hyperfibrinolysis detected by rotational thromboelastometry. (b) Restoration of normal fibrinolysis after antifibrinolytic treatment

Abbreviations: $\alpha=$ alpha angle; $\mathrm{A} 5=$ amplitude at 5 minutes after $\mathrm{CT} ; \mathrm{A} 10=$ amplitude at 10 minutes after $\mathrm{CT} ; \mathrm{CFT}=$ clot formation time; $C T=$ clotting time; $L I 30=$ Lysis index at 30 minutes after $C T ; M C F=$ maximum clot firmness; $M L=$ maximum lysis

underdiagnosed due to an absence of appropriate tests. ${ }^{1}$ Conventional coagulation tests, such as prothrombin time, activated partial thromboplastin time, and thrombin time, are ineffective in detecting hyperfibrinolysis. Viscoelastic haemostatic assays are the only tests for rapid detection and quantification of hyperfibrinolysis. ${ }^{2}$

Rotational thromboelastometry is a form of viscoelastic assay. It provides a global haemostatic assessment from initial platelet activation, through platelet aggregation, clot strengthening by crosslinked fibrin, to clot dissolution. The degree of clot lysis is expressed numerically as maximum lysis. Hyperfibrinolysis is diagnosed when maximum lysis exceeds $15 \%$ within a 60 -minute ROTEM analysis. Schöchl et $\mathrm{al}^{3}$ further quantified this condition based on the time course of clot dissolution, as reflected by the lysis index at 30 minutes and at 60 minutes. They categorised complete clot lysis within 30 minutes as fulminant hyperfibrinolysis; intermediate when complete clot lysis was within 30 to 60 minutes; and late when complete clot lysis exceeded 60 minutes. Prompt recognition of hyperfibrinolysis by ROTEM analysis guides appropriate antifibrinolytic therapy. Untreated hyperfibrinolysis has been shown to be associated with refractory bleeding and increased mortality. ${ }^{1}$

Rotational thromboelastometry has multiple advantages over conventional coagulation tests. In contrast to conventional coagulation tests that use plasma, ROTEM uses whole blood and can determine the contribution of both cellular and 
plasma components of haemostasis. Conventional coagulation tests provide only a quantitative assessment of individual procoagulation factors; results are not necessarily a good indication of the in vivo haemostatic process, especially in patients with chronic liver disease. ${ }^{4}$ Rotational thromboelastometry also provides real-time functional assessment of the haemostatic process, from clot formation to its lysis, and is useful for identifying hyperfibrinolysis. There is evidence that use of ROTEM-guided haemostatic strategy reduces transfusion requirement in different clinical settings. ${ }^{5-7}$

Understanding its limitations is equally important in the interpretation of ROTEM analysis. It is insensitive to the effect of platelet inhibitors such as aspirin and clopidogrel. Thrombin, the strongest activator of platelets, is produced in large amounts during ROTEM analysis, masking the inhibitory effects of antiplatelet agents. ${ }^{8}$ It is also poor at detecting conditions that affect platelet adhesion and aggregation, with Von Willebrand's disease being a well-known example. ${ }^{9}$ The ROTEM analysis is undertaken at $37^{\circ} \mathrm{C}$ to mimic physiological conditions so the negative effect of hypothermia on coagulation is not reflected by the analysis. ${ }^{9}$

In conclusion, owing to the limitations of conventional coagulation tests, hyperfibrinolysis is frequently underdiagnosed. With its ability of realtime functional haemostatic assessment from clot formation to lysis, ROTEM analysis allows better insight into the complex haemocoagulation process. The rapid detection of hyperfibrinolysis by ROTEM can guide prompt antifibrinolytic treatment, possibly reducing the transfusion requirement in bleeding patients.

\section{Author contributions}

Concept or design: KM Kwok, KL Lee, SY Lam, T Liong, KI Law.

Acquisition of data: KM Kwok.

Analysis or interpretation of data: KM Kwok, KL Lee, SY Lam, T Liong, KI Law.

Drafting of the manuscript: KM Kwok.

Critical revision of the manuscript for important intellectual content: All authors.

\section{Conflicts of interest}

The authors have no conflicts of interest to disclose.

\section{Funding/support}

This study received no specific grant from any funding agency in the public, commercial, or not-for-profit sectors.

\section{Ethics approval}

The patient was treated in accordance with the Declaration of Helsinki. Patient was incapable to provide informed consent for publication.

\section{References}

1. Theusinger OM, Wanner GA, Emmert MY, et al. Hyperfibrinolysis diagnosed by rotational thromboelastometry (ROTEM) is associated with higher mortality in patients with severe trauma. Anesth Analg 2011;113:1003-12.

2. Yeung MC, Tong SY, Tong PY, Cheung BH, Ng JY, Leung GK. Use of viscoelastic haemostatic assay in emergency and elective surgery. Hong Kong Med J 2015;21:45-51.

3. Schöchl H, Frietsch T, Pavelka M, Jámbor C. Hyperfibrinolysis after major trauma: differential diagnosis of lysis patterns and prognostic value of thrombelastometry. J Trauma 2009;67:125-31.

4. Tripodi A, Mannucci PM. The coagulopathy of chronic liver disease. N Engl J Med 2011;365:147-56.

5. De Pietri L, Bianchini $M$, Montalti $R$, et al Thrombelastography-guided blood product use before invasive procedures in cirrhosis with severe coagulopathy: a randomized, controlled trial. Hepatology 2016;63:566-73.

6. Veigas PV, Callum J, Rizoli S, Nascimento B, da Luz LT. A systematic review on the rotational thrombelastometry $\left(\right.$ ROTEM $\left.^{\circledR}\right)$ values for the diagnosis of coagulopathy, prediction and guidance of blood transfusion and prediction of mortality in trauma patients. Scand J Trauma Resusc Emerg Med 2016;24:114.

7. Vymazal T, Astraverkhava M, Durila M. Rotational thromboelastometry helps to reduce blood product consumption in critically ill patients during small surgical procedures at the intensive care unit-a retrospective clinical analysis and literature search. Transfus Med Hemother 2018;45:385-7.

8. Lang $\mathrm{T}$, von Depka M. Possibilities and limitations of thrombelastometry/-graphy [in German]. Hamostaseologie 2006;26(3 Suppl 1):S20-9.

9. Srivastava A, Kelleher A. Point-of-care coagulation testing. Continuing Educ Anaesthesia Crit Care Pain 2013;13:12-6. 